\title{
Management of Mikania micrantha in Young Robusta Coffee Plantation of Karbi Anglong District of Assam
}

\author{
A.R. Bora ${ }^{1 *}$, J. Deka ${ }^{2}$, I.C. Barua ${ }^{2}$ and B. Barman ${ }^{1}$ \\ ${ }^{1}$ Regional Coffee Research Station, Coffee Board, Diphu, Assam- 782 460, India \\ ${ }^{2}$ Assam Agricultural University, Jorhat, Assam- 785 013, India \\ *Corresponding author
}

\section{Keywords}

Mikania micrantha, Robusta Coffee, Karbi Anglong,

Article Info

Accepted:

10 January 2019

Available Online:

10 February 2019

\section{A B S T R A C T}

A field experiment was conducted at the Regional Coffee Research Station, Diphu, Assam during 2016 and 2017 for effective management of Mikania micrantha in young robusta coffee plantation. The experiment was laid out in factorial RBD with 3 replications of 9 treatments consisting of 3 gibberellic acid concentrations viz., $0 \mathrm{ppm}, 250 \mathrm{ppm}$ and 500 $\mathrm{ppm}$ and 3 weed management practices viz., Oxyfluorfen $(0.29 \mathrm{~kg} / \mathrm{ha})$ followed by Glyphosate $(0.99 \mathrm{~kg} / \mathrm{ha})$, Oxyfluorfen $(0.29 \mathrm{~kg} / \mathrm{ha})$ followed by Glyphosate $(0.99 \mathrm{~kg} / \mathrm{ha})+$ $2,4-\mathrm{D}(0.73 \mathrm{~kg} / \mathrm{ha})$ and weedy check. During both the years, gibberellic acid $500 \mathrm{ppm}$ recorded significantly higher density and dry weight of $M$. micrantha at 40 days after gibberellic acid application (DAGA) compared to other treatments, but different gibberellic acid treatments were at par in respect of Micania density and dry weight at 80, 120 and 160 DAGA as well as growth parameters of young coffee. In herbicide treatments, there was no $M$. micrantha during the entire period of experimentation. Application of Oxyfluorfen $(0.29 \mathrm{~kg} / \mathrm{ha})$ followed by Glyphosate $(0.99 \mathrm{~kg} / \mathrm{ha})$ and Oxyfluorfen $(0.29 \mathrm{~kg} / \mathrm{ha})$ followed by Glyphosate $(0.99 \mathrm{~kg} / \mathrm{ha})+2,4-\mathrm{D} \quad(0.73 \mathrm{~kg} / \mathrm{ha})$ resulted significantly higher growth parameters of young coffee as compared to weedy check in both the years but the herbicide treatments were at par. The hormone and herbicide application did not interact significantly in respect of density and dry weight of $M$. micrantha at 80,120 and 160 DAGA. It was concluded that M. micrantha could be managed effectively by application of Oxyfluorfen $\left(0.29 \mathrm{~kg} \mathrm{ha}^{-1}\right)$ followed by Glyphosate $\left(0.99 \mathrm{~kg} \mathrm{ha}^{-1}\right)$.

\section{Introduction}

Mikania micrantha is a perennial climbing vine and it is listed as one of the world's 32 worst invasive plants (Lowe et al., 2000). It is native to Central and South America (Holm et al., 1977) and widely distributed in the tropics and subtropics (Xie et al., 2010). M. micrantha climbs up to the top of the plant canopy and creates a dense cover which damages or kills other plants by blocking light (Holm et al., 1977). Further, it competes for water and soil nutrient and releases substances into soil that inhibit the growth of other plants (Huang et al., 2000, Li and Jin, 2010). 
There are more than 100 species of coffee, out of these only two are commercially cultivated viz. Coffea arabica L. (Arabica type coffee) and Coffea canephora Pierre (Robusta type coffee). In Karbi Anglong district of Assam, robusta coffee is predominantly cultivated. $M$. micrantha is one of the most problematic weeds in robusta coffee plantations of Karbi Anglong district of Assam as the climatic conditions of the district are favourable for the weed. However, there is no recommended practice for management of $M$. micrantha in young coffee plantations. So, the study was undertaken in order to develop suitable agrotechnologies for its management.

\section{Materials and Methods}

The study was carried out during 2016 and 2017 at the Regional Coffee Research Station (RCRS), Diphu, Assam located at $25^{\circ} 92^{\prime} \mathrm{N}$ latitude and $93^{\circ} 44^{\prime} \mathrm{E}$ longitude and altitude of $170 \mathrm{~m}$ above mean sea level.

During both the years, the weekly mean maximum temperature ranged from $20.7^{\circ} \mathrm{C}$ to $35.3^{\circ} \mathrm{C}$ whereas weekly mean minimum temperature ranged from $6.2^{\circ} \mathrm{C}$ to $28.3^{\circ}$. The weekly average relative humidity ranged from 85 to 96 per cent during the morning hours, while in the evening hours it ranged from 47 to 82.9 per cent. The total rainfall received during 2016 was $907.2 \mathrm{~mm}$ in 16 rainy days whereas it was $756.2 \mathrm{~mm}$ in 21 rainy days during 2017. The soil of the experimental site was sandy loam with $\mathrm{pH}$ value of 5.12.

The experiment was laid out in factorial randomised block design with 3 replications of 9 treatments consisting of 3 gibberellic acid concentrations viz., 0 ppm, 250 ppm and 500 ppm and 3 weed management practices viz., Oxyfluorfen $(0.29 \mathrm{~kg} / \mathrm{ha})$ followed by Glyphosate $(0.99 \mathrm{~kg} / \mathrm{ha})$ at $5-10 \mathrm{~cm}$ weed height, Oxyfluorfen $(0.29 \mathrm{~kg} / \mathrm{ha})$ followed by Glyphosate $(0.99 \mathrm{~kg} / \mathrm{ha})+2,4-\mathrm{D}(0.73 \mathrm{~kg} / \mathrm{ha})$ at $5-10 \mathrm{~cm}$ height of weed and weedy check. Gibberellic Acid (GA) was applied on the soil surface after removal of all the weeds from the plot. Oxyfluorfen $\left(0.29 \mathrm{~kg} \mathrm{ha}^{-1}\right)$ was applied on the soil surface 20 days after GA application after thoroughly cleaning the soil surface. Glyphosate $\left(0.99 \mathrm{~kg} \mathrm{ha}^{-1}\right)$ and 2,4-D $\left(0.73 \mathrm{~kg} \mathrm{ha}^{-1}\right)$ were applied 80 days after Oxyfluorfen application when the weeds attained about $5-10 \mathrm{~cm}$ height. Pits of size 45 $\mathrm{cm} \times 45 \mathrm{~cm} \mathrm{x} 45 \mathrm{~cm}$ were dug in the month of April, 2016 and exposed for weathering for 15 to 20 days. After that, the pits were filled with the surrounding top soil with the addition of $2 \mathrm{~kg}$ FYM and $30 \mathrm{~g}$ rock phosphate. Vigorous and disease free Coffea canephora selection 3R (CxR) saplings with six pair of leaves were planted in the field in the month of July, 2016.

Agricultural lime @ 2.5 tonne per hectare was applied during the month of November, in the second year of cultivation. The recommended dose of fertilizer @ 38:28:38 gram of $\mathrm{N}, \mathrm{P}_{2} \mathrm{O}_{5}$ and $\mathrm{K}_{2} \mathrm{O}$ per sapling in the form of urea $(46 \%$ $\mathrm{N})$, rock phosphate $\left(20 \% \mathrm{P}_{2} \mathrm{O}_{5}\right)$ and muriate of potash $\left(60 \% \quad \mathrm{~K}_{2} \mathrm{O}\right)$ was applied in two equal splits in the month of April and September. The coffee saplings were allowed to grow up to $1.2 \mathrm{~m}$ height. When the saplings reached the recommended height, the vertically growing main stem was cut $5 \mathrm{~cm}$ above the node near the recommended topping height of $1.2 \mathrm{~m}$.

\section{Results and Discussion}

\section{M. micrantha density and dry weight in the field}

During both the years of experimentation, application of GA 500 ppm recorded significantly higher $M$. micrantha density and dry weight at 40 days after gibberellic acid application (DAGA) compared to GA 250 ppm and no GA application (Table 1 and 4). 
At 80,120 and 160 DAGA, there was no significant difference in $M$. micrantha density and dry weight in different GA concentrations during both the years of experimentation (Table 2 and 5). This might be attributed to elimination of $M$. micrantha from the field due to application of herbicides in the field at 20 and 100 DAGA. In both the years of experimentation, significantly higher $M$. micrantha density and dry weight was recorded in weedy plots at 40, 80, 120 and 160 DAGA.

In herbicide treatments i.e. Oxyfluorfen $\left(0.29 \mathrm{~kg} \mathrm{ha}^{-1}\right)$ followed by Glyphosate $(0.99$ $\left.\mathrm{kg} \mathrm{ha}{ }^{-1}\right)$ and Oxyfluorfen (0.29kg ha-1) followed by Glyphosate $\left(0.99 \mathrm{~kg} \mathrm{ha}^{-1}\right)+2,4-$ $\mathrm{D}\left(0.73 \mathrm{~kg} \mathrm{ha}^{-1}\right), M$. micrantha density was nil. This might be due to effective control of $M$. micrantha by the herbicides.

In both the years of experimentation, a significant interaction effect of hormone and herbicide application on density at 40 DAGA (Table 3 and 6) was resulted due to GA and weed management treatments and the lowest values of $M$. micrantha density were observed with different GA levels combined with herbicide treatments. This could be due to the fact that M. micrantha was eliminated by the effect of herbicides and GA was effective only up to 40 DAGA.

Table.1 Density of $M$. micrantha in young coffee at 40 and 80 DAGA as influenced by hormone and herbicide application

\begin{tabular}{|c|c|c|c|c|}
\hline \multirow[t]{3}{*}{ Treatments } & \multicolumn{4}{|c|}{ M. micrantha density (No. $\mathrm{m}^{-2}$ ) } \\
\hline & \multicolumn{2}{|c|}{40 DAGA } & \multicolumn{2}{|c|}{80 DAGA } \\
\hline & 2016 & 2017 & 2016 & 2017 \\
\hline \multicolumn{5}{|l|}{ Hormone application $(\mathrm{H})$} \\
\hline $\mathrm{H}_{1}$ : Without GA & $\begin{array}{c}1.03 \\
(0.78)\end{array}$ & $\begin{array}{c}1.06 \\
(0.89)\end{array}$ & $\begin{array}{c}1.9 \\
(1.89)\end{array}$ & $\begin{array}{c}1.27 \\
(1.78)\end{array}$ \\
\hline $\mathrm{H}_{2}$ : With GA 250 ppm & $\begin{array}{c}1.20 \\
(1.44)\end{array}$ & $\begin{array}{c}1.20 \\
(1.44)\end{array}$ & $\begin{array}{c}1.34 \\
(2.11)\end{array}$ & $\begin{array}{c}1.30 \\
(1.89)\end{array}$ \\
\hline $\mathrm{H}_{3}$ : With GA 500 ppm & $\begin{array}{c}1.38 \\
(2.33)\end{array}$ & $\begin{array}{c}1.34 \\
(2.11)\end{array}$ & $\begin{array}{c}1.34 \\
(2.11)\end{array}$ & $\begin{array}{c}1.34 \\
(2.11)\end{array}$ \\
\hline $\operatorname{SEM}( \pm)$ & 0.04 & 0.04 & 0.05 & 0.05 \\
\hline $\mathrm{CD}(\mathrm{P}=\mathbf{0 . 0 5})$ & 0.13 & 0.11 & NS & NS \\
\hline \multicolumn{5}{|l|}{ Herbicide application (G) } \\
\hline $\begin{array}{l}\mathrm{G}_{1} \text { : Oxyfluorfen }\left(0.29 \mathrm{~kg} \mathrm{ha}^{-1}\right) \\
\text { fb Glyphosate }\left(0.99 \mathrm{~kg} \mathrm{ha}^{-1}\right)\end{array}$ & $\begin{array}{c}0.71 \\
(0.00)\end{array}$ & $\begin{array}{c}0.71 \\
(0.00)\end{array}$ & $\begin{array}{c}0.71 \\
(0.00)\end{array}$ & $\begin{array}{c}0.71 \\
(0.00)\end{array}$ \\
\hline $\begin{array}{l}\mathrm{G}_{2} \text { : Oxyfluorfen }\left(0.29 \mathrm{~kg} \mathrm{ha}^{-1}\right) \\
\text { fb. Glyphosate }\left(0.99 \mathrm{~kg} \mathrm{ha}^{-1}\right) \\
+2,4-\mathrm{D}\left(0.73 \mathrm{~kg} \mathrm{ha}^{-1}\right)\end{array}$ & $\begin{array}{c}0.71 \\
(0.00)\end{array}$ & $\begin{array}{c}0.71 \\
(0.00)\end{array}$ & $\begin{array}{c}0.71 \\
(0.00)\end{array}$ & $\begin{array}{c}0.71 \\
(0.00)\end{array}$ \\
\hline$G_{3}$ : Weedy & $\begin{array}{c}2.20 \\
(4.56)\end{array}$ & $\begin{array}{c}2.19 \\
(4.44)\end{array}$ & $\begin{array}{c}2.56 \\
(6.11)\end{array}$ & $\begin{array}{c}2.50 \\
(5.78)\end{array}$ \\
\hline $\operatorname{SEM}( \pm)$ & 0.04 & 0.04 & 0.05 & \\
\hline $\mathrm{CD}(\mathrm{P}=\mathbf{0 . 0 5})$ & 0.13 & 0.11 & 0.14 & 0.14 \\
\hline Interaction (HxG) & $\mathrm{S}$ & $\mathrm{S}$ & NS & NS \\
\hline $\mathrm{CV}(\%)$ & 10.49 & 9.39 & 10.42 & 10.46 \\
\hline
\end{tabular}

GA - Gibberellic acid, DAGA - Days after gibberellic acid application, fb- followed by.

Figures in parenthesis are mean of original values. Data subject to square root transformation. 
Table.2 Density of M. micrantha in young coffee at 120 and 160 DAGA as influenced by hormone and herbicide application

\begin{tabular}{|c|c|c|c|c|}
\hline \multirow[t]{3}{*}{ Treatments } & \multicolumn{4}{|c|}{ M. micrantha density (No. $\mathrm{m}^{-2}$ ) } \\
\hline & \multicolumn{2}{|c|}{120 DAGA } & \multicolumn{2}{|c|}{160 DAGA } \\
\hline & 2016 & 2017 & 2016 & 2017 \\
\hline \multicolumn{5}{|l|}{ Hormone application (H) } \\
\hline $\mathrm{H}_{1}$ : Without GA & $1.25(1.67)$ & $1.20(1.44)$ & $1.12(1.11)$ & $1.18(1.33)$ \\
\hline $\mathrm{H}_{2}$ : With GA $250 \mathrm{ppm}$ & $1.27(1.78)$ & $1.25(1.67)$ & $1.15(1.22)$ & $1.20(1.44)$ \\
\hline $\mathrm{H}_{3}$ : With GA 500 ppm & $1.30(1.89)$ & $1.25(1.67)$ & $1.15(1.22)$ & $1.20(1.44)$ \\
\hline $\operatorname{SEM}( \pm)$ & 0.07 & 0.05 & 0.04 & 0.04 \\
\hline $\mathrm{CD}(\mathrm{P}=\mathbf{0 . 0 5})$ & NS & NS & NS & NS \\
\hline \multicolumn{5}{|l|}{ Herbicide application (G) } \\
\hline $\begin{array}{l}\mathrm{G}_{1} \text { : Oxyfluorfen }\left(0.29 \mathrm{~kg} \mathrm{ha}^{-1}\right) \mathrm{fb} \\
\text { Glyphosate }\left(0.99 \mathrm{~kg} \mathrm{ha}^{-1}\right)\end{array}$ & $0.71(0.00)$ & $0.71(0.00)$ & $0.71(0.00)$ & $0.71(0.00)$ \\
\hline $\begin{array}{l}\mathrm{G}_{2} \text { : Oxyfluorfen }\left(0.29 \mathrm{~kg} \mathrm{ha}^{-1}\right) \text { fb. } \\
\text { Glyphosate }\left(0.99 \mathrm{~kg} \mathrm{ha}^{-1}\right)+2,4-\mathrm{D} \\
\left(0.73 \mathrm{~kg} \mathrm{ha}^{-1}\right)\end{array}$ & $0.71(0.00)$ & $0.71(0.00)$ & $0.71(0.00)$ & $0.71(0.00)$ \\
\hline G3: Weedy & $2.41(5.33)$ & $2.29(4.78)$ & $2.01(3.56)$ & $2.16(4.22)$ \\
\hline $\operatorname{SEM}( \pm)$ & 0.07 & 0.05 & 0.04 & 0.04 \\
\hline $\mathrm{CD}(\mathrm{P}=\mathbf{0 . 0 5})$ & 0.15 & 0.15 & 0.12 & 0.13 \\
\hline Interaction (HxG) & NS & NS & NS & NS \\
\hline CV (\%) & 11.80 & 11.82 & 10.50 & 11.24 \\
\hline
\end{tabular}

GA - Gibberellic acid, DAGA - Days after gibberellic acid application, fb- followed by.

Figures in parenthesis are mean of original values. Data subject to square root transformation.

Table.3 Density of $M$. micrantha $\left(\right.$ Nos. $\mathrm{m}^{-2}$ ) in young coffee at 40 DAGA due to interaction of hormone and herbicide application in 2016 and 2017

\begin{tabular}{|c|c|c|c|c|c|c|}
\hline \multirow{3}{*}{$\begin{array}{l}\text { Hormone } \\
\text { application }\end{array}$} & \multicolumn{6}{|c|}{ Herbicide application } \\
\hline & \multicolumn{2}{|c|}{$\begin{array}{c}\mathrm{G}_{1}: \text { Oxyfluorfen fb } \\
\text { Glyphosate }\end{array}$} & \multicolumn{2}{|c|}{$\begin{array}{c}\mathrm{G}_{2} \text { : Oxyfluorfen fb Glyphosate } \\
+2,4-D\end{array}$} & \multicolumn{2}{|c|}{$G_{3}:$ Weedy } \\
\hline & 2016 & 2017 & 2016 & 2017 & 2016 & 2017 \\
\hline $\mathrm{H}_{1}$ : Without GA & 0.71 & 0.71 & 0.71 & 0.71 & 1.68 & 1.77 \\
\hline $\begin{array}{l}\mathrm{H}_{2}: \text { With GA } 250 \\
\text { ppm }\end{array}$ & 0.71 & 0.71 & 0.71 & 0.71 & 2.19 & 2.19 \\
\hline $\begin{array}{l}\mathrm{H}_{3}: \text { With GA } 500 \\
\text { ppm }\end{array}$ & 0.71 & 0.71 & 0.71 & 0.71 & 2.73 & 2.61 \\
\hline $\operatorname{SEM}( \pm)$ & 0.07 & 0.07 & & & & \\
\hline $\mathrm{CD}(\mathrm{P}=0.05)$ & 0.22 & 0.20 & & & & \\
\hline $\mathrm{CV}(\%)$ & 10.49 & 9.39 & & & & \\
\hline
\end{tabular}

GA - Gibberellic acid, DAGA - Days after gibberellic acid application 
Table.4 Dry weight of $M$. micrantha in young coffee at 40 and 80 DAGA as influenced by hormone and herbicide application

\begin{tabular}{|c|c|c|c|c|}
\hline \multirow[t]{3}{*}{ Treatments } & \multicolumn{4}{|c|}{ M. micrantha dry weight $\left(\mathrm{g} \mathrm{m}^{-2}\right)$} \\
\hline & \multicolumn{2}{|c|}{40 DAGA } & \multicolumn{2}{|c|}{ 80 DAGA } \\
\hline & 2016 & 2017 & 2016 & 2017 \\
\hline \multicolumn{5}{|l|}{ Hormone application (H) } \\
\hline $\mathrm{H}_{1}$ : Without GA & $0.80(0.15)$ & $0.81(0.18)$ & $1.09(0.98)$ & $1.06(0.90)$ \\
\hline $\mathrm{H}_{2}$ : With GA 250 ppm & $0.86(0.30)$ & $0.84(0.25)$ & $1.11(1.07)$ & $1.09(0.97)$ \\
\hline $\mathrm{H}_{3}$ : With GA 500 ppm & $0.92(0.44)$ & $0.91(0.41)$ & $1.11(1.06)$ & $1.10(1.02)$ \\
\hline $\operatorname{SEM}( \pm)$ & 0.02 & 0.02 & 0.03 & 0.04 \\
\hline $\mathrm{CD}(\mathrm{P}=\mathbf{0 . 0 5})$ & 0.05 & 0.05 & NS & NS \\
\hline \multicolumn{5}{|l|}{ Herbicide application (G) } \\
\hline $\begin{array}{l}\mathrm{G}_{1} \text { : Oxyfluorfen }\left(0.29 \mathrm{~kg} \mathrm{ha}^{-1}\right) \text { fb Glyphosate }(0.99 \\
\left.\mathrm{kg} \mathrm{ha}^{-1}\right)\end{array}$ & $0.71(0.00)$ & $0.71(0.00)$ & $0.71(0.00)$ & $0.71(0.00)$ \\
\hline $\begin{array}{l}\mathrm{G}_{2} \text { : Oxyfluorfen }\left(0.29 \mathrm{~kg} \mathrm{ha}^{-1}\right) \text { fb. Glyphosate } \\
\left(0.99 \mathrm{~kg} \mathrm{ha}^{-1}\right)+2,4-\mathrm{D}\left(0.73 \mathrm{~kg} \mathrm{ha}^{-1}\right)\end{array}$ & $0.71(0.00)$ & $0.71(0.00)$ & $0.71(0.00)$ & $0.71(0.00)$ \\
\hline$G_{3}:$ Weedy & $1.17(0.89)$ & $1.15(0.84)$ & $1.89(3.10)$ & $1.84(2.89)$ \\
\hline $\operatorname{SEM}( \pm)$ & 0.02 & 0.02 & 0.03 & 0.04 \\
\hline $\mathrm{CD}(\mathrm{P}=\mathbf{0 . 0 5})$ & 0.05 & 0.05 & 0.09 & 0.11 \\
\hline Interaction (HxG) & $\mathrm{S}$ & $\mathrm{S}$ & NS & NS \\
\hline CV (\%) & 5.29 & 6.15 & 7.96 & 10.48 \\
\hline
\end{tabular}

GA - Gibberellic acid, DAGA - Days after gibberellic acid application, fb- followed by.

Figures in parenthesis are mean of original values. Data subject to square root transformation.

Table.5 Dry weight of $M$. micrantha in young coffee at 120 and 160 DAGA as influenced by hormone and herbicide application

\begin{tabular}{|c|c|c|c|c|}
\hline \multirow[t]{3}{*}{ Treatments } & \multicolumn{4}{|c|}{ M. micrantha dry weight $\left(\mathrm{g} \mathrm{m}^{-2}\right)$} \\
\hline & \multicolumn{2}{|c|}{120 DAGA } & \multicolumn{2}{|c|}{160 DAGA } \\
\hline & 2016 & 2017 & 2016 & 2017 \\
\hline \multicolumn{5}{|l|}{ Hormone application $(\mathrm{H})$} \\
\hline $\mathrm{H}_{1}:$ Without GA & $1.32(2.01)$ & $1.27(1.77)$ & $1.46(2.79)$ & $1.54(3.28)$ \\
\hline $\mathrm{H}_{2}$ : With GA 250 ppm & $1.34(2.15)$ & $1.31(2.01)$ & $1.50(3.04)$ & $1.59(3.65)$ \\
\hline $\mathrm{H}_{3}$ : With GA 500 ppm & $1.37(2.27)$ & $1.32(2.01)$ & $1.51(3.11)$ & $1.59(3.59)$ \\
\hline $\operatorname{SEM}( \pm)$ & 0.05 & 0.05 & 0.07 & 0.07 \\
\hline $\mathrm{CD}(\mathrm{P}=\mathbf{0 . 0 5})$ & NS & NS & NS & NS \\
\hline \multicolumn{5}{|l|}{ Herbicide application (G) } \\
\hline $\begin{array}{l}\mathrm{G}_{1} \text { : Oxyfluorfen }\left(0.29 \mathrm{~kg} \mathrm{ha}^{-1}\right) \text { fb Glyphosate } \\
\left(0.99 \mathrm{~kg} \mathrm{ha}^{-1}\right)\end{array}$ & $0.71(0.00)$ & $0.71(0.00)$ & $0.71(0.00)$ & $0.71(0.00)$ \\
\hline $\begin{array}{l}\mathrm{G}_{2} \text { : Oxyfluorfen }\left(0.29 \mathrm{~kg} \mathrm{ha}^{-1}\right) \text { fb. Glyphosate } \\
\left(0.99 \mathrm{~kg} \mathrm{ha}^{-1}\right)+2,4-\mathrm{D}\left(0.73 \mathrm{~kg} \mathrm{ha}^{-1}\right)\end{array}$ & $0.71(0.00)$ & $0.71(0.00)$ & $0.71(0.00)$ & $0.71(0.00)$ \\
\hline$G_{3}$ : Weedy & $2.62(6.42)$ & $2.50(5.79)$ & $3.06(8.95)$ & $3.30(10.52)$ \\
\hline $\operatorname{SEM}( \pm)$ & 0.05 & 0.05 & 0.07 & 0.07 \\
\hline $\mathrm{CD}(\mathrm{P}=\mathbf{0 . 0 5})$ & 0.16 & 0.16 & 0.20 & 0.22 \\
\hline Interaction (HxG) & NS & NS & NS & NS \\
\hline CV (\%) & 12.16 & 12.19 & 13.65 & 13.89 \\
\hline
\end{tabular}

GA - Gibberellic acid, DAGA - Days after gibberellic acid application, fb-followed by.

Figures in parenthesis are mean of original values. Data subject to square root transformation. 
Table.6 Dry weight of $M$. micrantha $\left(\mathrm{g} \mathrm{m}^{-2}\right)$ in young coffee at 40 DAGA under interaction of hormone and herbicide application in 2016 and 2017

\begin{tabular}{|c|c|c|c|c|c|c|}
\hline \multirow{3}{*}{$\begin{array}{l}\text { Hormone } \\
\text { application }\end{array}$} & \multicolumn{6}{|c|}{ Herbicide application } \\
\hline & \multicolumn{2}{|c|}{$\begin{array}{l}\mathrm{G}_{1}: \text { Oxyfluorfen fb } \\
\text { Glyphosate }\end{array}$} & \multicolumn{2}{|c|}{$\begin{array}{c}\mathrm{G}_{2} \text { : Oxyfluorfen fb Glyphosate } \\
+2,4-D\end{array}$} & \multicolumn{2}{|c|}{ G $_{3}$ : Weedy } \\
\hline & 2016 & 2017 & 2016 & 2017 & 2016 & 2017 \\
\hline $\mathrm{H}_{1}$ : Without GA & 0.71 & 0.71 & 0.71 & 0.71 & 0.98 & 1.02 \\
\hline $\begin{array}{l}\mathrm{H}_{2} \text { : With GA } 250 \\
\text { ppm }\end{array}$ & 0.71 & 0.71 & 0.71 & 0.71 & 1.18 & 1.12 \\
\hline $\begin{array}{l}\mathrm{H}_{3}: \text { With GA } 500 \\
\text { ppm }\end{array}$ & 0.71 & 0.71 & 0.71 & 0.71 & 1.35 & 1.31 \\
\hline $\operatorname{SEM}( \pm)$ & 0.03 & 0.04 & & & & \\
\hline $\mathrm{CD}(\mathrm{P}=\mathbf{0 . 0 5})$ & 0.08 & 0.09 & & & & \\
\hline CV $(\%)$ & 5.29 & 6.15 & & & & \\
\hline
\end{tabular}

GA - Gibberellic acid, DAGA - Days after gibberellic acid application.

Table.7 Sapling height $(\mathrm{cm})$ of young coffee at different days after planting as influenced by hormone and herbicide application

\begin{tabular}{|c|c|c|c|c|}
\hline \multirow[t]{2}{*}{ Treatments } & \multicolumn{2}{|c|}{90 DAP } & \multicolumn{2}{|c|}{180 DAP } \\
\hline & 2016 & 2017 & 2016 & 2017 \\
\hline \multicolumn{5}{|l|}{ Hormone application (H) } \\
\hline $\mathrm{H}_{1}$ : Without GA & 22.07 & 90.84 & 30.28 & 96.18 \\
\hline $\mathrm{H}_{2}$ : With GA 250 ppm & 27.26 & 93.22 & 30.36 & 98.78 \\
\hline $\mathrm{H}_{3}$ : With GA 500 ppm & 27.08 & 84.34 & 30.57 & 99.51 \\
\hline $\operatorname{SEM}( \pm)$ & 0.86 & 5.74 & 1.03 & 5.02 \\
\hline $\mathrm{CD}(\mathrm{P}=0.05)$ & NS & NS & NS & NS \\
\hline \multicolumn{5}{|l|}{ Herbicide application (G) } \\
\hline $\begin{array}{l}\mathrm{G}_{1}: \text { Oxyfluorfen }(0.29 \mathrm{~kg} / \mathrm{ha}) \mathrm{fb} \\
\text { Glyphosate }(0.99 \mathrm{~kg} / \mathrm{ha})\end{array}$ & 28.41 & 99.91 & 32.39 & 103.56 \\
\hline $\begin{array}{l}\mathrm{G}_{2} \text { : Oxyfluorfen }(0.29 \mathrm{~kg} / \mathrm{ha}) \mathrm{fb} \text {. } \\
\text { Glyphosate }(0.99 \mathrm{~kg} / \mathrm{ha})+2,4- \\
D(0.73 \mathrm{~kg} / \mathrm{ha})\end{array}$ & 27.96 & 100.00 & 31.91 & 106.70 \\
\hline G3: Weedy & 25.01 & 67.51 & 26.90 & 84.21 \\
\hline $\operatorname{SEM}( \pm)$ & 0.86 & 5.74 & 1.03 & 5.02 \\
\hline $\mathrm{CD}(\mathrm{P}=0.05)$ & 2.59 & 17.20 & 3.09 & 15.05 \\
\hline Interaction (HxG) & NS & NS & NS & NS \\
\hline CV $(\%)$ & 9.55 & 19.24 & 10.16 & 15.34 \\
\hline
\end{tabular}

GA - Gibberellic acid, DAGA - Days after gibberellic acid application, fb- followed by.

Figures in parenthesis are mean of original values. Data subject to square root transformation. 
Table.8 Sapling girth $(\mathrm{cm})$ of young coffee at different days after planting as influenced by hormone and herbicide application

\begin{tabular}{|c|c|c|c|c|}
\hline \multirow[t]{2}{*}{ Treatments } & \multicolumn{2}{|c|}{90 DAP } & \multicolumn{2}{|c|}{180 DAP } \\
\hline & 2016 & 2017 & 2016 & 2017 \\
\hline \multicolumn{5}{|l|}{ Hormone application (H) } \\
\hline $\mathrm{H}_{1}$ : Without GA & 0.8 & 4.34 & 1.21 & 4.89 \\
\hline $\mathrm{H}_{2}$ : With GA 250 ppm & 0.93 & 4.28 & 1.18 & 4.84 \\
\hline $\mathrm{H}_{3}$ : With GA 500 ppm & 0.97 & 4.48 & 1.21 & 5.20 \\
\hline $\operatorname{SEM}( \pm)$ & 0.06 & 0.21 & 0.08 & 0.31 \\
\hline $\mathrm{CD}(\mathrm{P}=0.05)$ & NS & NS & NS & NS \\
\hline \multicolumn{5}{|l|}{ Herbicide application (G) } \\
\hline $\begin{array}{l}\mathrm{G}_{1} \text { : Oxyfluorfen }(0.29 \mathrm{~kg} / \mathrm{ha}) \text { fb Glyphosate }(0.99 \\
\mathrm{kg} / \mathrm{ha})\end{array}$ & 1.08 & 4.62 & 1.38 & 5.57 \\
\hline $\begin{array}{l}\mathrm{G}_{2} \text { : Oxyfluorfen }(0.29 \mathrm{~kg} / \mathrm{ha}) \text { fb. Glyphosate }(0.99 \\
\mathrm{kg} / \mathrm{ha})+2,4-D(0.73 \mathrm{~kg} / \mathrm{ha})\end{array}$ & 1.10 & 4.98 & 1.40 & 5.76 \\
\hline$G_{3}$ : Weedy & 0.70 & 3.50 & 0.82 & 3.61 \\
\hline $\operatorname{SEM}( \pm)$ & 0.06 & 0.21 & 0.08 & 0.31 \\
\hline $\mathrm{CD}(\mathrm{P}=0.05)$ & 0.18 & 0.64 & 0.23 & 0.92 \\
\hline Interaction (HxG) & NS & NS & NS & NS \\
\hline CV $(\%)$ & 19.26 & 14.70 & 19.59 & 18.51 \\
\hline
\end{tabular}

GA - Gibberellic acid, DAGA - Days after gibberellic acid application, fb- followed by.

Figures in parenthesis are mean of original values. Data subject to square root transformation.

Table.9 Leaf number (Nos.) of young coffee at different days after planting as influenced by hormone and herbicide application

\begin{tabular}{|c|c|c|c|c|}
\hline \multirow[t]{2}{*}{ Treatments } & \multicolumn{2}{|c|}{90 DAP } & \multicolumn{2}{|c|}{180 DAP } \\
\hline & 2016 & 2017 & 2016 & 2017 \\
\hline \multicolumn{5}{|l|}{ Hormone application (H) } \\
\hline $\mathrm{H}_{1}$ : Without GA & 16.13 & 53.03 & 18.30 & 64.58 \\
\hline $\mathrm{H}_{2}$ : With GA 250 ppm & 16.18 & 50.13 & 18.93 & 60.03 \\
\hline $\mathrm{H}_{3}$ : With GA 500 ppm & 16.27 & 56.52 & 19.06 & 68.40 \\
\hline $\operatorname{SEM}( \pm)$ & 0.82 & 3.47 & 0.76 & 4.03 \\
\hline $\mathrm{CD}(\mathrm{P}=0.05)$ & NS & NS & NS & NS \\
\hline \multicolumn{5}{|l|}{ Herbicide application (G) } \\
\hline $\begin{array}{l}\mathrm{G}_{1} \text { : Oxyfluorfen }(0.29 \mathrm{~kg} / \mathrm{ha}) \text { fb Glyphosate }(0.99 \\
\mathrm{kg} / \mathrm{ha})\end{array}$ & 17.19 & 64.04 & 20.54 & 77.91 \\
\hline $\begin{array}{l}\mathrm{G}_{2} \text { : Oxyfluorfen }(0.29 \mathrm{~kg} / \mathrm{ha}) \text { fb. Glyphosate }(0.99 \\
\mathrm{kg} / \mathrm{ha})+2,4-\mathrm{D}(0.73 \mathrm{~kg} / \mathrm{ha})\end{array}$ & 17.22 & 67.72 & 20.93 & 80.01 \\
\hline$G_{3}$ : Weedy & 14.27 & 27.92 & 14.81 & 35.09 \\
\hline $\operatorname{SEM}( \pm)$ & 0.82 & 3.47 & 0.76 & 4.03 \\
\hline $\mathrm{CD}(\mathrm{P}=0.05)$ & 2.45 & 10.42 & 2.29 & 12.09 \\
\hline Interaction (HxG) & NS & NS & NS & NS \\
\hline CV $(\%)$ & 15.14 & 19.58 & 12.21 & 18.81 \\
\hline
\end{tabular}

GA - Gibberellic acid, DAGA - Days after gibberellic acid application, fb- followed by.

Figures in parenthesis are mean of original values. Data subject to square root transformation. 
Table.10 Leaf area $\left(\mathrm{cm}^{2}\right)$ of young coffee at different days after planting as influenced by hormone and herbicide application

\begin{tabular}{|c|c|c|c|c|}
\hline \multirow[t]{2}{*}{ Treatments } & \multicolumn{2}{|c|}{90 DAP } & \multicolumn{2}{|c|}{180 DAP } \\
\hline & 2016 & 2017 & 2016 & 2017 \\
\hline \multicolumn{5}{|l|}{ Hormone application (H) } \\
\hline $\mathrm{H}_{1}$ : Without GA & 1206.89 & 8034.55 & 1555.69 & 10235.58 \\
\hline $\mathrm{H}_{2}$ : With GA 250 ppm & 1209.63 & 7595.20 & 1609.98 & 9515.28 \\
\hline $\mathrm{H}_{3}$ : With GA 500 ppm & 1216.85 & 8563.12 & 1634.13 & 10841.40 \\
\hline $\operatorname{SEM}( \pm)$ & 61.28 & 528.01 & 65.57 & 641.21 \\
\hline $\mathrm{CD}(\mathrm{P}=0.05)$ & NS & NS & NS & NS \\
\hline \multicolumn{5}{|l|}{ Herbicide application (G) } \\
\hline $\begin{array}{l}\mathrm{G}_{1} \text { : Oxyfluorfen }(0.29 \mathrm{~kg} / \mathrm{ha}) \text { fb Glyphosate }(0.99 \\
\mathrm{kg} / \mathrm{ha})\end{array}$ & 1278.27 & 9702.73 & 1780.58 & 12348.91 \\
\hline $\begin{array}{l}\mathrm{G}_{2} \text { : Oxyfluorfen }(0.29 \mathrm{~kg} / \mathrm{ha}) \text { fb. Glyphosate }(0.99 \\
\mathrm{kg} / \mathrm{ha})+2,4-D(0.73 \mathrm{~kg} / \mathrm{ha})\end{array}$ & 1289.38 & 10259.92 & 1789.90 & 12681.76 \\
\hline$G_{3}$ : Weedy & 1065.72 & 4230.22 & 1229.32 & 5561.59 \\
\hline $\operatorname{SEM}( \pm)$ & 61.28 & 528.01 & 65.57 & 641.21 \\
\hline $\mathrm{CD}(\mathrm{P}=\mathbf{0 . 0 5})$ & 183.27 & 1578.32 & 195.99 & 1916.53 \\
\hline Interaction (HxG) & NS & NS & NS & NS \\
\hline CV (\%) & 15.14 & 19.58 & 12.26 & 18.81 \\
\hline
\end{tabular}

GA - Gibberellic acid, DAGA - Days after gibberellic acid application, fb- followed by.

Figures in parenthesis are mean of original values. Data subject to square root transformation.

Table.11 Number of primary branch (Nos.) in young coffee at different days after planting as influenced by hormone and herbicide application

\begin{tabular}{|c|c|c|c|c|}
\hline \multirow[t]{2}{*}{ Treatments } & \multicolumn{2}{|c|}{90 DAP } & \multicolumn{2}{|c|}{180 DAP } \\
\hline & 2016 & 2017 & 2016 & 2017 \\
\hline \multicolumn{5}{|l|}{ Hormone application (H) } \\
\hline $\mathrm{H}_{1}$ : Without GA & 0 & 5.16 & 0 & 7.84 \\
\hline $\mathrm{H}_{2}$ : With GA 250 ppm & 0 & 5.09 & 0 & 7.62 \\
\hline $\mathrm{H}_{3}$ : With GA 500 ppm & 0 & 5.38 & 0 & 7.92 \\
\hline $\operatorname{SEM}( \pm)$ & & 0.33 & & 0.50 \\
\hline $\mathrm{CD}(\mathrm{P}=\mathbf{0 . 0 5})$ & & NS & & NS \\
\hline \multicolumn{5}{|l|}{ Herbicide application (G) } \\
\hline $\begin{array}{l}\mathrm{G}_{1}: \text { Oxyfluorfen }(0.29 \mathrm{~kg} / \mathrm{ha}) \text { fb Glyphosate }(0.99 \\
\mathrm{kg} / \mathrm{ha})\end{array}$ & 0 & 6.33 & 00 & 9.20 \\
\hline $\begin{array}{l}\mathrm{G}_{2} \text { : Oxyfluorfen }(0.29 \mathrm{~kg} / \mathrm{ha}) \text { fb. Glyphosate }(0.99 \\
\mathrm{kg} / \mathrm{ha})+2,4-\mathrm{D}(0.73 \mathrm{~kg} / \mathrm{ha})\end{array}$ & 0 & 6.62 & 00 & 9.27 \\
\hline$G_{3}$ : Weedy & 0 & 2.67 & 00 & 4.92 \\
\hline $\operatorname{SEM}( \pm)$ & & 0.33 & & 0.50 \\
\hline $\mathrm{CD}(\mathrm{P}=\mathbf{0 . 0 5})$ & & 0.97 & & 1.51 \\
\hline Interaction (HxG) & & NS & & NS \\
\hline CV $(\%)$ & & 18.72 & & 19.36 \\
\hline
\end{tabular}

GA - Gibberellic acid, DAGA - Days after gibberellic acid application, fb- followed by.

Figures in parenthesis are mean of original values. Data subject to square root transformation. 
Table.12 Mortality (\%) of young coffee at different years as influenced by hormone and herbicide application

\begin{tabular}{|c|c|c|}
\hline Treatments & 2016 & 2017 \\
\hline $\begin{array}{l}\mathrm{H}_{1} \mathrm{G}_{1}: \text { Without GA; Oxyfluorfen }(0.29 \mathrm{~kg} / \mathrm{ha}) \text { fb Glyphosate } \\
(0.99 \mathrm{~kg} / \mathrm{ha})\end{array}$ & 0 & 0 \\
\hline $\begin{aligned} \mathrm{H}_{1} \mathrm{G}_{2}: & \text { Without GA; Oxyfluorfen }(0.29 \mathrm{~kg} / \mathrm{ha}) \mathrm{fb} . \\
& \text { Glyphosate }(0.99 \mathrm{~kg} / \mathrm{ha})+2,4-\mathrm{D}(0.73 \mathrm{~kg} / \mathrm{ha})\end{aligned}$ & 0 & 0 \\
\hline$H_{1} G_{3}$ : Without GA; Weedy & 20.00 & 23.20 \\
\hline $\begin{array}{l}\mathrm{H}_{2} \mathrm{G}_{1}: \text { With GA } 250 \mathrm{ppm} \text {; Oxyfluorfen }(0.29 \mathrm{~kg} / \mathrm{ha}) \mathrm{fb} \\
\text { Glyphosate }(0.99 \mathrm{~kg} / \mathrm{ha})\end{array}$ & 0 & 0 \\
\hline $\begin{aligned} & \mathrm{H}_{2} \mathrm{G}_{2}: \text { With GA } 250 \mathrm{ppm} \text {; Oxyfluorfen }(0.29 \mathrm{~kg} / \mathrm{ha}) \mathrm{fb} . \\
& \text { Glyphosate }(0.99 \mathrm{~kg} / \mathrm{ha})+2,4-\mathrm{D}(0.73 \mathrm{~kg} / \mathrm{ha})\end{aligned}$ & 0 & 0 \\
\hline $\mathrm{H}_{2} \mathrm{G}_{3}$ : With GA 250 ppm; Weedy & 22.00 & 24.00 \\
\hline $\begin{array}{l}\mathrm{H}_{3} \mathrm{G}_{1}: \text { With GA } 500 \mathrm{ppm} \text {; Oxyfluorfen }(0.29 \mathrm{~kg} / \mathrm{ha}) \mathrm{fb} \\
\text { Glyphosate }(0.99 \mathrm{~kg} / \mathrm{ha})\end{array}$ & 0 & 0 \\
\hline 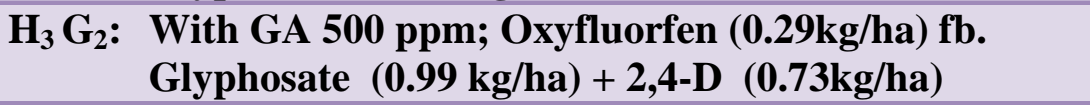 & 0 & 0 \\
\hline $\mathrm{H}_{3} \mathrm{G}_{3}$ : With GA 500 ppm; Weedy & 25.00 & 25.68 \\
\hline
\end{tabular}

GA-Gibberellic acid

\section{Growth of young coffee}

The growth parameters like sapling height, sapling girth, leaf number, leaf area and number of primary branches at 90 and 180 days after planting (DAP) were statistically at par among GA treatments in both the years (Table 7, 8, 9,10 and 11). The effect of GA application was visible up to 40 DAGA only, thereafter it disappeared. This might be the reason that no effect of soil applied GA was observed on sapling height, sapling girth, leaf number, leaf area and number of primary branches. Weed management practices recorded significant effect on growth of young coffee saplings at 90 and 180 DAP in both the years. Application of Oxyfluorfen $\left(0.29 \mathrm{~kg} \mathrm{ha}^{-1}\right)$ followed by Glyphosate $(0.99$ $\left.\mathrm{kg} \mathrm{ha}{ }^{-1}\right)$ and Oxyfluorfen (0.29kg ha $\left.{ }^{-1}\right)$ followed by Glyphosate $\left(0.99 \mathrm{~kg} \mathrm{ha}^{-1}\right)+2,4-$ $\mathrm{D}\left(0.73 \mathrm{~kg} \mathrm{ha}^{-1}\right)$ resulted significantly higher sapling height, sapling girth, leaf number, leaf area and number of primary branches of young coffee than that of control. Both the herbicide application treatments were at par in both the years in regard to growth of young coffee in 2016 and 2017. Effective control of all weeds by the sequential application of herbicides as compared to weedy check might have resulted better growth of young coffee plants.

\section{Mortality}

The data on mortality of young coffee is presented in Table 12. In both 2016 and 2017, mortality was observed only in weedy plots whereas no mortality of coffee saplings was recorded in herbicide treatments. In herbicide treated plots, weeds were completely eliminated due to the effect of herbicides. The mortality of coffee saplings in control plots might be due to adverse effect of $M$. micrantha.

In the present investigation, the treatment combinations of without GA and Oxyfluorfen $\left(0.29 \mathrm{~kg} \mathrm{ha}^{-1}\right)$ followed by Glyphosate $(0.99$ $\left.\mathrm{kg} \mathrm{ha}^{-1}\right)+2,4 \mathrm{D}\left(0.73 \mathrm{~kg} \mathrm{ha}^{-1}\right)$ as well as combination of without GA and Oxyfluorfen 
$\left(0.29 \mathrm{~kg} \mathrm{ha}^{-1}\right)$ followed by Glyphosate $(0.99$ $\mathrm{kg} \mathrm{ha}^{-1}$ ) showed best result in terms of $M$. micrantha control and higher growth parameters of young coffee. Therefore, it could be concluded that effective management of $M$. micrantha can be done by application of Oxyfluorfen $\left(0.29 \mathrm{~kg} \mathrm{ha}^{-1}\right) \mathrm{fb}$ Glyphosate $\left(0.99 \mathrm{~kg} \mathrm{ha}^{-1}\right)$.

\section{References}

Holm, L.G., Plucknett D.L., Pancho, J.K. and Herberger, J.P. 1977. The world's worst weeds: distribution and biology. University Press of Hawaii, Honolulu. Pp. 609.

Huang, Z.L., Cao, H.L., Liang, X.D., Ye, W.H., Feng, H.L. and Cai, C.X. 2000. The growth and damaging effect of Mikania micrantha in different habitats.
Journal of Tropical and Subtropical Botany 8: 131-138.

Li, J.M., Jin, Z.X. 2010. Potential allelopathic effects of Mikania micrantha on the seed germination and seedling growth of Coix lacryma-jobi. Weed Biol Manage 10(3):194-201.

Lowe, S., Browne, M., Boudjelas, S. and Depoorter, M. 2000. 100 of the World's Worst Invasive Alien Species. A selection from the Global Invasive Species Database. IUCN/SSC Invasive species specialist Group (ISSG), Auckland, New Zealand.

Xie, L. J., Zeng, R.S., BI, H. H., Song, Y.Y., Wangi, R. L., Su, Y.J., Chen,M., Chen, S. and Liu, Y.H. 2010. Allelochemical mediated invasion of exotic plants in China. Allelopathy Journal 25 (1): 3150 .

\section{How to cite this article:}

Bora, A.R., J. Deka, I.C. Barua and Barman, B. 2019. Management of Mikania micrantha in Young Robusta Coffee Plantation of Karbi Anglong District of Assam. Int.J.Curr.Microbiol.App.Sci. 8(03): 1004-1013. doi: https://doi.org/10.20546/ijcmas.2019.803.122 